\title{
VLSI Macromodeling and Signal Integrity Analysis via Digital Signal Processing Techniques
}

\author{
Chi-Un Lei, K.L. Man and Y. Wu
}

\begin{abstract}
Linear macromodeling has been applied to highfrequency circuit simulations to accelerate the global interconnect system simulation process. By approximating tabulated structure response data, reduced macromodels can be generated. However, conventional macromodeling approaches suffer from numerical robustness and convergence problems. This paper aims to apply digital signal processing techniques to facilitate the macromodeling process. Besides improving the existing widely adopted framework (called VFz) through introducing a robust discrete-time domain ( $z$-domain) computation, alternative macromodeling methodology (called VISA) has also been developed, which significantly simplifies the computation procedure. Furthermore, universal pre-processing technique (frequency warping) is introduced for a numerically favorable computation of the macromodeling process. These techniques have been shown to significantly improve the robustness and convergence of the modeling process.
\end{abstract}

Index Terms-Signal/Power Integrity, Interconnect/Package Modeling and Simulation, Digital Signal Processing, DiscreteTime Domain.

\section{INTRODUCTION}

W ITH the increasing operation frequency and decreasing feature size of very-large-scale integration (VLSI) circuits, high-frequency effects, such as signal delay, crosstalk and simultaneous switching noise, have become a dominant factor limiting integrated circuit (IC) system performance. Accurate and efficient simulation is required during the IC design phase to capture the high-frequency behaviours of electronic systems. Linear macromodeling, in this context, refers to replacing a high-order system by a small-order linear model with similar input-output responses, for computationally efficient simulation and timecritical design. Macromodels can be generated by fitting tabulated data from measurement/simulation, as shown in Fig. 1. There is a number of stringent modeling constraints in the non-linear computation for high-frequency and/or large scale systems systems, such as accuracy, computation complexity, manual intervention and numerical robustness. Some macromodeling approaches have been developed only very recently. In particular, Vector Fitting (VF) is regarded as a robust and simple broadband macromodeling technique and widely adopted in the signal integrity community. However, it suffers from convergence problem in the iterative calculation framework with initial pole assignment. Due to the strict requirements of the modeling problem and development

C.-U. Lei is with the Department of Electrical and Electronic Engineering, The University of Hong Kong, Hong Kong. Email: culei@eee.hku.hk, leonleihku@gmail.com

K.L. Man is with the Department of Computer Science and Software Engineering, Xi'an Jiaotong-Liverpool University, China. Email: ka.man@xjtlu.edu.cn

$\mathrm{Y}$. Wu is with the Department of Electrical and Electronic Engineering, Xi'an Jiaotong-Liverpool University, China. Email: yanyan.wu@xjtlu.edu.cn

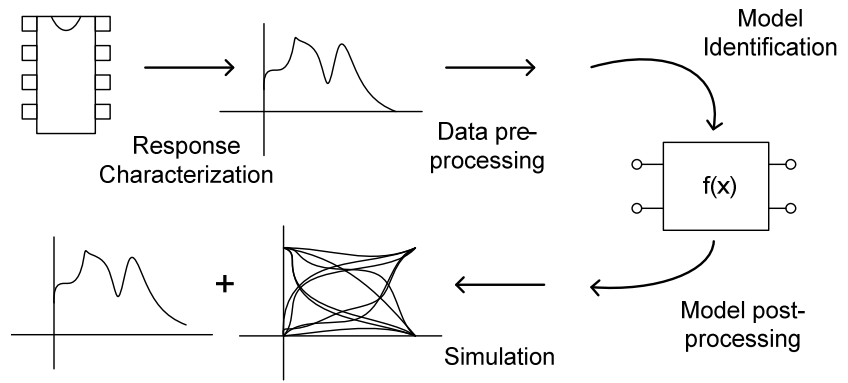

Fig. 1. Common Macromodeling Flow in the Signal Integrity Process.

of emerging technologies, there is no optimal algorithm so far, making macromodeling a challenging problem and a high value research topic. Furthermore, pre- and postprocessing techniques and improvements from non-controltheoretic perspectives have been less explored.

With "sampled response is a sampled and discretized signal sequence" as the fundamental concept, this research study [1] explores the feasibility and benefits of applying DSP techniques to the macromodeling process, focusing on:

1) improving the functionality and automation of the approximation process;

2) increasing the fitting accuracy of the approximation process;

3) reducing the computation time of the macromodeling procedure.

In this research study, we have developed new methodologies, generalizations of existing methodologies and pre-/postprocessing techniques to achieve the research outcomes. We have compared their performances with existing methodologies using industrial benchmark case studies. Such innovative application of DSP techniques has opened up new research frontiers for advancing the macromodeling process and VLSI circuit simulation.

\section{Proposed Solutions}

A. Macromodeling Process Development in the Vector Fitting Framework [2]

To build up a substantial foundation for the future development of the macromodeling process, we have explored the macromodeling process in signal/power integrity analyses in a system identification perspective. We have studied the major development of macromodeling process, especially development based on the Vector Fitting (VF) framework, along three directions: data, algorithms and models.

\section{B. Macromodeling Framework Advancements Via Discrete} Time Domain Computation [2]-[7]

To enhance the functionality of the widely adopted, iterative based Vector Fitting framework, we have proposed 
a family of improvements based on the root of the $z$ domain computation. First, we have proposed the $z$-domain counterpart of VF (VFz) [3], which uses $z$-domain partial fraction basis to seek a rational approximation to the desired response, namely,

$$
\hat{f}(z)=\left(\sum_{b=1}^{B} \frac{c_{b}}{1-z^{-1} a_{b}}\right)+d \approx f(z),
$$

where $B$ is the number of basis. This improves the numerical conditioning and convergence in broadband frequencysampled system macromodeling. Second, we have extended $\mathrm{VFz}$ to its discrete time-domain (TD-VFz) [5] variants for the specific time domain macromodeling purpose. TD- $\mathrm{VFz}$ does not require discretizing the continuous-time convolution integrals for each iteration and it provides a priori model order selection and a time delay extraction as pre-processing steps. Third, we have also proposed the discrete-time hybriddomain (HD-VFz) variants [6], which model responses with a better hybrid-domain accuracy through providing extra informative data. Fourth, in order to improve the funcationality of VF, we have extended VF to the effective asymmetrical response modeling of complex-domain systems (e.g., complex filter) by relaxing the complex conjugate pole pair restriction in VF [4] and VFz. Fifth, we have developed a versatile macromodeling adoption through a $P$-norm approximation expansion [2]. At last, we have modeled various case studies via $\mathrm{VFz}$ to demonstrate its excellent performance.

\section{VISA: Multiport Macromodeling Technique without Eigenvalue Computation [8], [9]}

To avoid numerically sensitive initial guess and expensive computation in time-domain macromodeling process, we have developd a rational function macromodeling algorithm called Versatile Impulse Structure Approximation (VISA). The idea is to regard the system response as the impulse response of a finite-impulse-response (FIR) filter, and then apply infinite-impulse-response (IIR) filter approximation techniques to generate the macromodel. By applying the idea of Walsh theorem and complementary signal, this approach can be interpreted as an interpolation problem. The optimal numerator polynomial can be determined with respect to a given denominator polynomial $Q(z)$. The interpolation can be achieved by minimizing the error term $\Delta(z)$ (a cascade of a causal FIR filter $R(z)$ and an allpass filter $A(z)$ ),

$$
\Delta(z)=A(z) z^{-1} R(z)=\frac{z^{-M} Q\left(z^{-1}\right)}{Q(z)} z^{-1} R(z) .
$$

By applying the idea of complementary signal, $Q(z)$ can be found without pole extraction (eigenvalue computation). This approach can be interpreted as a non-pole-based SteiglitzMcBride (SM) iteration without initial guess and eigenvalue computation. It can be solved iteratively using overdetermined equations solving and linear convolutions, which is numerically simple (i.e., robust and efficient) to determine macromodel parameters.

\section{Macromodeling Process Enhancement via Frequency Warping [9]}

To alleviate ill-conditioned computation problem in the linear-structure macromodeling process, we have proposed frequency warping, a spectral pre-processing scheme, by changing the response sampling distribution. Frequency warping transforms the structure response (time-/frequencysampled data), in order to introduce a numerically favorable fitting in the frequency domain, and to improve the fitting accuracy during the macromodeling process. The structure response is transformed by frequency warping which assigns an effective weighting in the frequency domain. When the response is warped, some frequency region of the response is stretched, while the others are compressed, which gives a higher resolution (accuracy) in the desired region and a lower resolution in other regions. The warping starts by replacing all operators $(s)$ in the original signal $G(s)$ by allpass operators $(\widetilde{s})$ in the warped signal $H(\widetilde{s})$ with a warping parameter $\gamma$

$$
G(s) \approx H(\widetilde{s}), \widetilde{s}=\frac{s+\gamma}{\gamma s+1},
$$

then continues the macromodeling process in $\widetilde{s}$ domain, finally warps the macromodel back to the $s$ (original) domain. We have also suggested that the frequency warping can be applied to multi-port configurations, different structure responses and algorithms through a simple bilinear transform.

\section{CONCLUSION}

In this paper, we have shown that the application of digital signal processing (DSP) techniques have significantly improved the robustness and efficiency of the macromodeling process. Such innovative application of DSP techniques has opened up new research frontiers for advancing the macromodeling process and VLSI circuit simulation. Results of this study can be extended to several interesting directions for future research, namely:

1) application of advanced DSP techniques to macromodeling and circuit simulation;

2) development of linear structure macromodeling using generalized descriptor systems;

3) development of non-linear structure macromodeling and circuit simulation;

4) optimization of macromodeling process for practical simulation.

\section{REFERENCES}

[1] C. U. Lei, "VLSI macromodeling and signal integrity analysis via digital signal processing techniques," Ph.D. dissertation, University of Hong Kong, Hong Kong, 2011.

[2] C. U. Lei, Y. Wang, Q. Chen, and N. Wong, "A decade of vector fitting development: Applications on signal/power integrity," in IAENG Transactions on Engineering Technologies (Vol.5). The American Institute of Physics, 2010, pp. 435-449.

[3] N. Wong and C. U. Lei, "IIR approximation of FIR filters via discretetime vector fitting," IEEE Trans. Signal Processing, vol. 56, no. 3, pp. 1296-1302, Mar. 2008.

[4] C. U. Lei, C. M. Cheung, H. K. Kwan, and N. Wong, "Efficient design of arbitrary complex response continuous-time IIR filter," in Trends in Communication Technologies and Engineering Science. Springer, 2009, pp. 163-176.

[5] C. U. Lei, H. K. Kwan, Y. Liu, and N. Wong, "Efficient linear macromodeling via least-squares response approximation," in Proc. IEEE Symp. Circuits and Systems, May 2008, pp. 2993-2996.

[6] C. U. Lei and N. Wong, "IIR approximation of FIR filter via discretetime hybrid-domain vector fitting," IEEE Signal Process. Lett., vol. 16, no. 6, pp. 533-537, Jun. 2009.

[7] — , "Linear marcomodeling framework advancements via discretetime domain computation," IEEE Trans. Adv. Packag, in preparation.

[8] —, "VISA: Versatile impulse structure approximation for timedomain linear macromodeling," in Proc. IEEE Asia and South Pacific Design Automation Conference, Jan. 2010, pp. 37-42.

[9] — , "WISE: Warped impulse structure estimation for time domain linear macromodeling," IEEE Trans. Adv. Packag, in preparation. 